\title{
On epidemic models with nonlinear cross-diffusion
}

\author{
$\underline{\text { S. Berres }}^{\mathrm{a}}$ and J. Gonzalez-Marin ${ }^{\mathrm{b}}$ \\ ${ }^{a}$ Departamento de Ciencias Matemáticas y Físicas, Universidad Católica de Temuco, Chile \\ ${ }^{\mathrm{b}}$ Departamento de Matemática, Universidade Federal da Bahia, Salvador, Brazil \\ Email: sberres@uct.cl
}

\begin{abstract}
Modelling and simulation of infectious diseases help to predict the likely outcome of an epidemic. A well-known generic model type for the quantitative description of the epidemic evolution dynamics by an ordinary differential equation is provided by so-called SIR models. These models classify a population into "suscepti-ble" (S), "infected" (I) and "recovered" (R) subgroups. One very early and simple prototype of an SIR-model is due to Kermack and McKendrick (1927). It describes the population evolution by the system of ordinary differential equations
\end{abstract}

$$
\frac{d S}{d t}=-\beta S I, \quad \frac{d I}{d t}=\beta S I-\gamma I, \quad \frac{d R}{d t}=\gamma I,
$$

with transmission rate $\beta>0$ and recovery rate $\gamma>0$. This equation can be written in general form as

$$
\frac{\partial S}{\partial t}=f(S, I), \quad \frac{\partial I}{\partial t}=g(S, I),
$$

where the epidemic interaction dynamics are modeled in the functions $f$ and $g$. The variable "R" can be dropped since its evolution is already implicit in the equations.

This basic SIR model can be extended by introducing a spatial distribution of both populations. In this contribution, it is shown how the spatial extension can be done by either a continuous or a discrete spatial distribution. For the continuous distribution, the model is typically formulated as a system of reaction-diffusion equations, where the reaction terms describe the local dynamics of susceptible and infected species, and the diffusion terms account for the spatial-distribution dynamics. For a discrete local distribution, the population is typically arranged in a series of patches. Each combination of population-type and patch corresponds to a particular variable. To describe the dynamics between different patches of the same population-type, the ordinary differential equations are extended by diffusion terms, which have an effect that is similar to the heat diffusion in Newton's law of cooling.

The dynamics are the same for continuous and discrete spatial distributions. At each location, both populations are present and interact locally according to an ordinary differential equation that governs the local epidemic interaction-dynamics. In addition, the spatial distribution allows us to model a spatial diffusion, which can be either "self-diffusion" or "cross-diffusion". For self-diffusion, the diffusion rate of each population depends on its particular local variation, whereas for cross-diffusion, the rate also depends on the local variation of the other population. For an SIR model, cross-diffusion is consistent with the phenomenon that the susceptible population avoids areas with an elevated percentage of the infected population.

It is known that diffusion in general and self-diffusion in particular aim towards an equilibrium solution, where the populations are homogeneously distributed. On the other hand, the coupling of diffusion with ordinary differential equations might provoke a nonequilibrium behavior, which is known as Turing instability. The extended SIR model provides a spatial pattern formation that is essentially driven by cross-diffusion.

In this contribution, nonlinear constitutive equations are suggested and analyzed for both self-diffusion and cross-diffusion. In particular, the modelling of cross-diffusion is reconsidered by starting from a basic SIR model, which is extended by either a discrete or continuous spatial distribution. Comparing the discrete model with patches to the continuous PDE model prepares the way for a micro-macro transition, where the continuous model can be deduced from the discrete. This deduction allows us to advance in the development of epidemic models with cross-diffusion. This is further elaborated in the complete version of the paper.

Keywords: Epidemic model, Cross-diffusion, Reaction-diffusion equation 


\section{INTRODUCTION}

This contribution is a further development of Berres and Ruiz-Baier (2011), where an epidemic model with nonlinear cross-diffusion is proposed and simulated by a multi-resolution scheme. Here, we propose an alternative modelling approach, which is based on a discrete model and a subsequent micro-macro transition; it is a preparation for the full paper version, where different model variants are discussed and a stability analysis of the discrete model with diffusion is carried out.

The two-dimensional reaction-diffusion system describing spatial epidemic dynamics with cross-diffusion is written as

$$
\begin{aligned}
S_{t} & =f(S, I)+\nabla \cdot(a(S) \nabla S)+\nabla \cdot(c(S, I) \nabla I), \\
I_{t} & =g(S, I)+\nabla \cdot(b(I) \nabla I),
\end{aligned}
$$

where $S=S(t, x)$ and $I=I(t, x)$ denote the populations of susceptible and infected persons, respectively, that move in the physical domain $(t, x) \in \Omega_{T}=(0, T) \times \Omega$. The self-diffusion rates $a(S), b(I)$ depend only on the individual populations types, whereas the cross-diffusion rate $c(S, I)$ depends on both population types. Since no immigration from outside is imposed, the Neumann boundary condition

$$
(a(S) \nabla S+c(S, I) \nabla I) \cdot n=0, \quad(b(I) \nabla S) \cdot n=0
$$

holds on the physical domain boundary $\partial \Omega$, where $n$ is the outer normal vector to the boundary. In the system (2) an additional equation for the recuperated population is omitted because the model does not consider their feedback on the susceptible or infected population.

The model enforces phase separation since the susceptible species avoid the infected by a cross-diffusion term $\nabla \cdot(c(S, I) \nabla I)$, see Sun et al. (2009), which is supposed to direct the flow in the opposite direction of the gradient $\nabla I$. The susceptible agents move away from the direction of the increasing gradient whenever there is a local increase in the infected population.

\subsection{Macro model by constitutive assumptions}

The macro model (2) can be closed by constitutive functions that reflect intuitions of the "real" behavior. Following the setting of Sun et al. (2009), in Berres and Ruiz-Baier (2011) first linear diffusion functions are simulated by a multi-resolution scheme, which refines the mesh at strong variations of the solution. For a linear diffusion the diffusion coefficients in (2) are chosen to be the constants

$$
a(S)=a_{0}, \quad b(I)=b_{0}, \quad c(S, I)=c_{0} .
$$

In order to demonstrate the versatility of the multi-resolution scheme and also to evolve the modelling variety, Berres and Ruiz-Baier (2011) proposed a nonlinear model variant of the parametric functions. There, the self-diffusion terms are chosen to have the form

$$
a(S)=a_{0} S^{m}, \quad b(I)=b_{0} I^{m} .
$$

The assumption that $m \in(-1,0)$ implies degressive growth since

$$
\partial_{S} a(S)<0, \quad \partial_{I} b(I)<0 \text { for all } S, I .
$$

The biological interpretation is that the tendency to avoid crowds reduces with higher numbers as the population "gets used" to them. For $m=0$ the functions (4) correspond to the linear model (3) .

A nonlinear cross-diffusion function $c(S, I)$ implements a situation-dependent tendency of the susceptible population to avoid the infected population. This behavior reflects an average psychological disposition. An approach to model such a disposition is as follows. From the perspective of the susceptible population, avoidance is pursued whenever there is a detectable fraction of the infected population. For a low percentage of the infected population, the awareness of the susceptible population has not matured or is temporarily inactive because there is no vital urgency for self-protection. In the other extreme, at large population numbers, such a selective detection is neither possible nor makes sense, since there is less, or even no chance to avoid infection in the crowd. The population number affects the conscious disposition of avoidance. Therefore, the 
cross-diffusion coefficient is designed to be negligible for both small and large number of populations. This is imposed by the following constraints

$$
\begin{aligned}
& c(S, 0)=0, \quad c(0, I)=0, \quad \text { for all } \quad S, I \in \mathbb{R}, \\
& c(S, I)=0 \quad \text { for } \quad I \geq V(S),
\end{aligned}
$$

where $V$ is a Lipschitz-continuous monotonically decreasing function with a zero for some $S>0$. For example, one might choose $V(S)=c_{1}-S$ with $c_{1}>0$. By these constraints, the reaction-diffusion equation (2) counts only with cross-diffusion inside the domain

$$
\tilde{\Omega}:=\{(S, I): S, I \geq 0, V(S) \leq I\}
$$

and degenerates into an equation without cross-diffusion outside the domain.

The constraints (5) are satisfied, for example, by the quadratic function

$$
c(S, I)= \begin{cases}c_{0} S I\left(c_{1}-S-I\right), \quad c_{0}, c_{1}>0, & \text { if } \quad S, I \geq 0, \quad S+I \leq c_{1}, \\ 0 & \text { otherwise },\end{cases}
$$

which is a convex function that takes its global maximum in

$$
(\hat{S}, \hat{I})=\left(\frac{c_{1}}{3}, \frac{c_{1}}{3}\right)
$$

In the sequel, some supporting arguments for the constraints (5) and in particular for the nonlinear model (6) are summarized. First of all, the constraint $c(S, 0)=0$ for all $S$ corresponds to a carefree state. When there is little or no infection, people are not sufficiently aware of the danger of the disease, even though there might be some single dangerous interactions. The constraint $c(0, I)=0$ for all $I$ is set not only for symmetry reasons; a few susceptible agents have little chance of sensing the importance of separating themselves from the infected population. Therefore, the susceptible population is trapped in the infection zone (which is characterized by its high percentage of infected population) and sooner or later infected. The maximum $(\hat{S}, \hat{I})$ implies that there is most avoidance when there is a fairly equal mixing of susceptible and infected populations. At small population numbers there is less need and at large total population numbers less possibility for avoidance. There is little possibility of selective avoidance when there is a large concentration of persons. Since the infected species is present anywhere and thus cannot be sustainably avoided in the crowd, there is little or no chance to keep distant from the infected species. Thus, fatalism rules above a certain threshold "upper" population number. This fatalism is modelled by the assumption that the cross-diffusion coefficient vanishes above a threshold population number. This upper population bound is set by the function $V$ such that $c_{1}$ corresponds to a maximum population, where, in the case that $c_{1}=u+v$, complete fatalism rules.

\section{MODELLING AND ANALYSIS OF THE INFECTION DYNAMICS}

A class of ordinary differential equations (1) modelling epidemics under consideration has the general structure

$$
\frac{\partial S}{\partial t}=\ell(S)-\beta(S, I), \quad \frac{\partial I}{\partial t}=\beta(S, I)-k(I),
$$

where $\ell(S)$ is a logistic function with the properties

$$
\ell(S) \begin{cases}>0 & \text { for } \quad S \in(0, L) \\ \leq 0 & \text { else }\end{cases}
$$

$ß(S, I)$ is the phase transition from susceptible to infected population with

$$
ß(S, I) \geq 0, \quad ß(0, \cdot)=0, \quad ß(\cdot, 0)=0, \quad \frac{\partial ß}{\partial S} \geq 0, \quad \frac{\partial ß}{\partial I} \geq 0,
$$

and $k(I) \geq 0$ is the recovery rate. An example of a specification of equation (7) is (see e.g. Sun et al. (2009))

$$
f(S, I)=r S(1-S / K)-\beta \frac{S I}{S+I}, \quad g(S, I)=\beta \frac{S I}{S+I}-k I,
$$


with the model parameters $K$ (the carrying capacity of the susceptible species), $r$ (the intrinsic birth rate), $\beta$ (the rate of disease transmission), and $k$ (the recovery rate of the infected species).

In the sequel, some properties of the equilibrium of the non-diffusive model (1), (8) are collected; see also Berres and Ruiz-Baier (2011). The equilibrium states are defined as pairs $(S, I)$ such that $f(S, I)=0$ and $g(S, I)=0$ hold simultaneously. For $(1),(8)$, the equilibrium points are $F_{s}=(K, 0)$, which corresponds to the disease-free point, and

$$
F_{c}=\left(S^{*}, I^{*}\right)=\left(\frac{K(k+r-\beta)}{r}, \frac{K(k+r-\beta)(\beta-k)}{r k}\right),
$$

which corresponds to an endemic stationary state. Note that the origin $(0,0)$ is a singular point and thus cannot be an equilibrium. The non-trivial equilibrium state $F_{c}$ is located in the first quadrant if

$$
k<\beta<k+r
$$

The inequality $\beta>k$ imposes that the transmission rate is greater than the recovery rate.

The system considered here is asymptotically stable in the sense that if the initial data are chosen close to the non-trivial equilibrium, the solution converges to this equilibrium. This can be shown analytically by demonstrating that the Jacobian matrix

$$
A=\left(\begin{array}{ll}
a_{11} & a_{12} \\
a_{21} & a_{22}
\end{array}\right)=\left(\begin{array}{ll}
\frac{\partial f}{\partial S}(S, I) & \frac{\partial f}{\partial I}(S, I) \\
\frac{\partial g}{\partial S}(S, I) & \frac{\partial g}{\partial I}(S, I)
\end{array}\right)
$$

has eigenvalues $\lambda_{1}, \lambda_{2}$ with negative real part. This can be checked by

$$
\operatorname{tr} A=\frac{\partial f}{\partial S}+\frac{\partial g}{\partial I}<0, \quad \operatorname{det} A=\frac{\partial f}{\partial S} \frac{\partial g}{\partial I}-\frac{\partial f}{\partial I} \frac{\partial g}{\partial S}>0
$$

Indeed, the Jacobian matrix corresponding to the specification (8) is

$$
A(S, I)=\left(\begin{array}{cc}
r\left(1-2 \frac{S}{K}\right)-\frac{\beta I^{2}}{(S+I)^{2}} & -\frac{\beta S^{2}}{(S+I)^{2}} \\
\frac{\beta v^{2}}{(u+v)^{2}} & \frac{\beta S^{2}}{(S+I)^{2}}-k
\end{array}\right),
$$

which is evaluated in the endemic equilibrium as

$$
A\left(S^{*}, I^{*}\right)=\left(\begin{array}{cc}
\beta-r-\frac{k^{2}}{\beta} & -\frac{k^{2}}{\beta} \\
\frac{(\beta-k)^{2}}{\beta} & \frac{k(k-\beta)}{\beta}
\end{array}\right) .
$$

It turns out to be independent of the parameter $K$. The endemic equilibrium $\left(S^{*}, I^{*}\right)$ is stable if

$$
\operatorname{det} A\left(S^{*}, I^{*}\right)=\frac{k(\beta-k)(k+r-\beta)}{\beta}>0, \quad \operatorname{tr} A\left(S^{*}, I^{*}\right)=\beta-r-k<0,
$$

which is valid exactly if (9) holds, i.e. if the equilibrium is located in the first quadrant. More precisely, the characteristic polynomial $\lambda^{2}-\lambda \operatorname{tr} A\left(S^{*}, I^{*}\right)+\operatorname{det} A\left(S^{*}, I^{*}\right)=0$ has roots

$$
\lambda_{1,2}\left(F_{c}\right)=\frac{1}{2}(\beta-k-r) \pm \frac{\sqrt{\beta(r+k-\beta)\left(r \beta+4 k^{2}-\beta^{2}-3 k \beta\right)}}{2 \beta},
$$

so $F_{c}$ is a complex center if

$$
r<\beta+3 k-4 \frac{k^{2}}{\beta}
$$


S. Berres and J. Gonzalez-Marin, On epidemic models with nonlinear cross-diffusion

The complex center $F_{c}$ is always an attractor since by (9) the real part of $\lambda_{1,2}\left(F_{c}\right)$ is always negative. Under the condition (9), for $F_{s}$ one gets

$$
\operatorname{det} A(K, 0)=r(k-\beta)<0, \quad \operatorname{tr} A(K, 0)=\beta-r-k<0 .
$$

The negative determinant indicates that $F_{s}$ is a saddle point. Indeed, one has the eigenvalues $\lambda_{1}=-r<0<$ $\beta-k=\lambda_{2}$. The negative eigenvalue has eigenvector $(1,0)^{\mathrm{T}}$, i.e. the trajectories pass on the $S$-axis towards the saddle point $F_{s}$ and move on towards the complex center $F_{c}$.

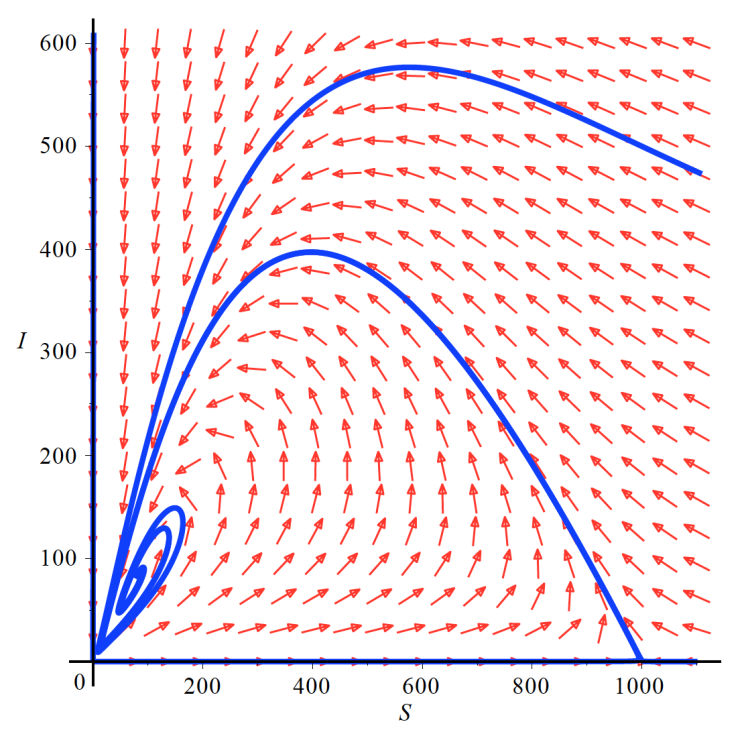

Figure 1. Phase portrait for the ODE system (1), (8) with the parameters $k=0.25, r=0.27, K=$ $1000, \beta=0.5$.

In Figure 1, the phase portrait of the ODE system (1), (8) is shown with several choices of initial data. In particular, the trajectory from the saddle point $F_{s}$ towards the complex center $F_{c}$ is drawn.

The preceding development can be summarized in the following theorem.

Theorem 1. If the endemic equilibrium $F_{c}$ is located in the first quadrant, i.e. inequality (9) is satisfied, and moreover if (10) holds, then $F_{c}$ is a complex center and $F_{s}$ is a saddle point located on the $S$-axis which has a trajectory from $F_{s}$ towards $F_{c}$.

\section{DISCRETE MODELS}

The ordinary differential equation (1) can be extended by introducing several patches (Aly and Farkas (2004a,b); Kiss and Kovács (2008)) as

$$
\begin{aligned}
\frac{\partial S_{1}}{\partial t}=f\left(S_{1}, I_{1}\right)+d_{S}\left(W\left(S_{2}, I_{2}\right)-W\left(S_{1}, I_{1}\right)\right), & \frac{\partial I_{1}}{\partial t}=g\left(S_{1}, I_{1}\right)+d_{I}\left(I_{2}-I_{1}\right), \\
\frac{\partial S_{2}}{\partial t}=f\left(S_{2}, I_{2}\right)+d_{S}\left(W\left(S_{1}, I_{1}\right)-W\left(S_{2}, I_{2}\right)\right), & \frac{\partial I_{2}}{\partial t}=g\left(S_{2}, I_{2}\right)+d_{I}\left(I_{1}-I_{2}\right),
\end{aligned}
$$

where $d_{S}$ and $d_{I}$ are constant diffusion rates and the subindices indicate the corresponding patches. Patches are different locations between which a population can migrate. There is no diffusion when $d_{S}=d_{I}=0$. Thus, the endemic equilibrium can be preserved in both patches.

The diffusion terms consider a one-way cross-diffusion, where both populations affect their particular migrations, but only the infected population affects the diffusivity of the susceptible population. For the infected 
populations $I_{1}, I_{2}$ there is only self-diffusion. For the evolution of the susceptible populations $S_{1}, S_{2}$, both self- and cross-diffusion apply if the diffusion function $W(S, I)$ depends on both population types.

Setting $W(S, I)=S$ would correspond to self-diffusion only. A first approach for a cross-diffusion is to model the diffusion term as a product $W(S, I)=S I$. A variant is to set a combination of self- and cross-diffusion like

$$
W(S, I)=S(1+I)
$$

Other variants of cross-diffusion settings and the corresponding stability analysis are discussed in the full version of this paper.

The patch model (11) can be extended from two to several patches. In the constellation of more than two attached patches, the middle patches are affected by both neighboring patches, whereas the patches placed at the boundary have the same coupling structure as in situation (11) with two patches:

$$
\begin{aligned}
\frac{\partial S_{1}}{\partial t} & =f\left(S_{1}, I_{1}\right)+d_{S}\left(W_{2}-W_{1}\right), \quad \frac{\partial I_{1}}{\partial t}=g\left(S_{1}, I_{1}\right)+d_{I}\left(I_{2}-I_{1}\right), \\
\frac{\partial S_{j}}{\partial t} & =f\left(S_{j}, I_{j}\right)+d_{S}\left(W_{j+1}-2 W_{j}+W_{j-1}\right), \quad j=2, \ldots, N-1, \\
\frac{\partial I_{j}}{\partial t} & =g\left(S_{j}, I_{j}\right)+d_{I}\left(I_{j+1}-2 I_{j}+I_{j-1}\right), \quad j=2, \ldots, N-1, \\
\frac{\partial S_{N}}{\partial t} & =f\left(S_{N}, I_{N}\right)+d_{S}\left(W_{N-1}-W_{N}\right), \quad \frac{\partial I_{N}}{\partial t}=g\left(S_{N}, I_{N}\right)+d_{I}\left(I_{N-1}-I_{N}\right),
\end{aligned}
$$

where $W_{j}=W\left(S_{j}, I_{j}\right), j=1, \ldots, N$ accounts for diffusive interaction. The patches with index $j=$ $2, \ldots, N-2$ can be referred to as the "middle patches" and the patches with index $j \in\{1, N\}$ mark the boundaries. The Jacobian matrix of the model (13) for general $N \geq 3$ can be written using the structure of a block matrices as

$$
M_{N}=\left(\begin{array}{cccccc}
A-D & D & 0 & \cdots & \cdots & 0 \\
D & A-2 D & D & \ddots & & \vdots \\
0 & D & A-2 D & \ddots & \ddots & \vdots \\
\vdots & \ddots & \ddots & \ddots & \ddots & 0 \\
\vdots & & \ddots & D & A-2 D & D \\
0 & \ldots & \ldots & 0 & D & A-D
\end{array}\right)
$$

A stability analysis for the situation of three patches is done in the full version.

\subsection{Micro-macro transition}

As an alternative to the constitutive modelling approach that leads to the cross-diffusion (6), the continuous spatial distribution can be deduced from the discrete model (13) by a micro-macro transition. Indeed, the system with discrete spatial distribution (13) can be transformed to a system with continuous spatial distribution by associating the patches to positions $S_{j}=S(j \Delta x), I_{j}=I(j \Delta x), j=0, \ldots, n, \Delta x=1 / N$. The interior approximations of $j=2, \ldots, n-1$ in the discrete model (13) are passing in the limit $h \rightarrow 0$ (and thus simultaneously $n \rightarrow \infty$ ) to the continuous distribution

$$
\lim _{h \rightarrow 0} \frac{\mathcal{X}(x+h)-2 \mathcal{X}(x)+\mathcal{X}(x-h)}{h^{2}}=\frac{\partial^{2}}{\partial x^{2}} \mathcal{X}(x) .
$$

With $\mathcal{X} \in\{W, I\}$, one gets

$$
\frac{\partial S}{\partial t}=f(S, I)+d_{S}\left(\partial_{x x} W\right), \quad \frac{\partial I}{\partial t}=g(S, I)+d_{I}\left(\partial_{x x} I\right) .
$$

Conversely, system (13) can be seen as discretization of (14). A second space-dimension could be handled analogously. In order to rewrite (14) in a quasi-linear form (2) one applies the chain rule to (12) giving

$$
\frac{\partial}{\partial x} S(x) I(x)=S I_{x}+I S_{x}
$$


In vector notation with variables $u=(S, I)^{\mathrm{T}}$, reaction term $F=(f, g)^{\mathrm{T}}$ and diffusion-reaction equation $u_{t}=F+\partial_{x} D \partial_{x} u$, this contributes to the diffusion matrix as

$$
\left(\begin{array}{cc}
1+I & S \\
0 & 0
\end{array}\right)\left(\begin{array}{l}
S_{x} \\
I_{x}
\end{array}\right)
$$

giving the diffusion matrix

$$
D=\left(\begin{array}{cc}
1+I & S \\
0 & I
\end{array}\right),
$$

which has an upper tridiagonal structure.

\section{CONCLUSION AND DISCUSSION}

The ODE model (1), (8) can be described as a Lotka-Volterra system with a ratio-dependent functional response and a logistic growth rate. Such models also have an interpretation in economic models (Balázsi and Kiss (2013), Farkas (1995), Farkas and Kotsis (1992)), where $S$ is interpreted as the number of free jobs, $I$ is the total labor force (those employed and those unemployed), $r$ is the natural per capita growth rate for free jobs, and $K$ the maximum number of jobs.

Several aspects will be developed in more detail in the full version of this paper. To start with, variants of cross-diffusion settings that are other than (12) are considered and the corresponding stability analysis is discussed. Then, a stability analysis for the the diffusion model (13) with several patches is performed, with special attention to the situation with three patches. Finally, the transition to the continuous spatial distribution of patches as formalized in model (14) is developed in more detail.

\section{ACKNOWLEDGEMENT}

The first author has been supported by Conicyt (Chile) through Fondecyt project \#1120587.

\section{REFERENCES}

Aly, S. and M. Farkas (2004a). Bifurcations in a predator-prey model in a patchy environment with diffusion. Nonlin. Anal. Real World Appl. 5, 519-526.

Aly, S. and M. Farkas (2004b). Competition in patchy environment with cross diffusion. Nonlin. Anal. Real World Appl. 5, 589-595.

Balázsi, L. and K. Kiss (2013). Crossdiffusion modeling in macroeconomics. Preprint (arXiv:1302.3958).

Berres, S. and R. Ruiz-Baier (2011). A fully adaptive numerical approximation for a two-dimensional epidemic model with nonlinear cross-diffusion. Nonlin. Anal. Real World Appl. 12 (5), 2888-2903.

Farkas, M. (1995). On the distribution of capital and labour in a closed economy. SEA Bull. Math. 19, 27-36.

Farkas, M. and M. Kotsis (1992). Predator-prey and wage-employment dynamics. Dyn. Econ. Mod. Opt. Contr., North-Holland, Amsterdam, 513-526.

Kermack, W. O. and A. G. McKendrick (1927). A contribution to the mathematical theory of epidemics. Proc. Roy. Soc. Lond. A 115, 700-721.

Kiss, K. and S. Kovács (2008). Qualitative behaviour of n-dimensional ratio-dependent predator-prey systems. Appl. Math. Comp. 199(2), 535-546.

Sun, G.-Q., Z. Jin, Q.-X. Liu, and L. Li (2009). Spatial pattern in an epidemic system with cross-diffusion of the susceptible. J. Biol. Syst. 17, 141-152. 